J. DIFFERENTIAL GEOMETRY

50 (1998) $387-413$

\title{
FEDOSOV CONNECTIONS ON KÄHLER SYMMETRIC MANIFOLDS AND TRACE DENSITY COMPUTATION
}

\author{
DMITRY E. TAMARKIN
}

\begin{abstract}
A Fedosov connection on a Kähler locally symmetric manifold in a compact explicit form was found. The trace density for the corresponding quantization was directly computed.
\end{abstract}

\section{Introduction}

In this paper we introduce a Fedosov connection on Kähler locally symmetric manifolds.

Contrary to the case of an arbitrary manifold, this connection appears to be written in a very compact form. A similar construction for an arbitrary Kähler manifold was independently elaborated in [3]. We also compute the trace density for this connection. (See Theorem 2.6). As a result of our computation, the trace density is expressed via geometrical data of the manifold. The final expression resembles a stationary phase approximation for some Feynman path integral. Easy calculation (see section 2) shows that this formula gives the same value as the index theorem for deformations [2], [4]. According to the result of the author [5] which says that any topological invariant on a symplectic manifold expressed in terms of curvature and symplectic form is a polynomial in characteristic classes and symplectic form, to prove the index theorem for deformations it suffices to prove it for Kähler symmetric manifolds. Thus, this yields a new proof of the index theorem for deformations that is parallel to Atiyah-Bott-Patody heat kernel proof.

Received February 12, 1998. 
Also, it would be interesting to generalize the formula for trace density obtained in this paper to arbitrary manifold and to reveal its physical sense.

Acknowledgments. The author would like to thank B.V. Fedosov, B.L. Tsygan, and R. Nest for the concern to this paper and for useful discussions. The author also greatly appreciates the work of referee whose very useful remarks helped a lot.

\subsection{Brief content of the paper}

In sections 1.1-1.3 we briefly review Fedosov quantization. Section 1.4 contains a collection of identities for the curvature tensor on Kähler locally symmetric manifolds that we need in the sequel. Section 1.5 gives a construction of a Fedosov connection on a locally symmetric Kähler manifold. In section 1.6 we quantize the Hamilton functions of symmetry transforms. In section 1.7 on our manifold, we inroduce Darboux coordinates, which are expressed in terms of the Hamilton functions of symmetry transforms.

In sections 2.1-2.2 we develop some technique for trace density computation, which is applied to the case of a locally symmetric Kähler manifold in section 2.3. In section 2.4 we apply the obtained formula for the trace density to $\mathbb{C} P^{n}$, and we get the same number as in the index theorem for deformations [2],[4].

\section{Deformation quantization on Kähler symmetric manifolds}

We will briefly describe B.V. Fedosov construction of deformation quantization [1] and construct a Fedosov connection on a symmetric Kähler manifold.

\subsection{Deformation quantization}

Let $M$ be a symplectic manifold. A deformation quantization on $M$ is an associative algebra denoted by $\mathbb{A}^{h}(M)$ whose elements are formal series from $C^{\infty}(M)[[h]]$. The additive operation is defined simply as the sum of functions, and the multiplication $*$ is supposed to satisfy the Correspondence principle

$$
f * g=f g-\frac{1}{2} i h\{f, g\}+o(h)
$$


and the Locality property

$$
f * g=\sum h^{i} D_{i}(f, g)
$$

where $D_{i}$ are bidifferential operators of finite order. The last condition means that the multiplication $*$ is well defined for $C^{\infty}$-jets at any point of $M$.

\subsection{The Weyl Algebra}

Let $V, \omega$ be a symplectic vector space. Define the Weyl algebra $\mathcal{W}(V)$ whose elements are formal series

$$
\sum_{\mu} a^{\mu} y^{\mu}
$$

where $\mu$ is a multiindex, $a^{\mu} \in \mathbb{C}[[h]]$, and $y^{1}, y^{2}, \ldots, y^{2 n}$ form a basis in $V$. The product $\circ$ is given by the formula

$$
a \circ b(x)=\left.\exp \left\{-i h \frac{\omega^{k j}}{2} \frac{\partial}{\partial y_{k}} \frac{\partial}{\partial z_{j}}\right\} a(y) b(z)\right|_{y=z=x},
$$

where $\omega^{i j}=<y^{i}, y^{j}>$, and $<,>$ is the symplectic scalar product on $V$. The Weyl algebra provides a deformation quantization for jets of functions on $V$.

A Symbol of an element $s(y) \in \mathcal{W}(V)$ is by definition its value at zero $\sigma(s)=s(0)$.

The Weyl algebra is naturally graded in the following way. Set $\operatorname{deg} h=2, \operatorname{deg} y=1$ for $y \in V$. The symplectic group $S p(V)$ acts on $\mathcal{W}(\mathcal{V})$ naturally preserving the grading and the symbol. This means that we can change the fiber in the symplectic bundle $T^{*} M$ from $\mathbb{R}^{2 n}$ to $\mathcal{W}\left(\mathbb{R}^{2 n}\right)$. Thus the new bundle of algebras is called the bundle of Weyl algebras $\mathcal{W}(M)$. Sections of this bundle form an algebra with the fiberwise addition and multiplication. The grading and the symbol of section of $\mathcal{W}(M)$ are also well defined fiberwise. Any element $f \in$ $C^{\infty}(M)[[h]]$ defines a section of $\mathcal{W}(M)$, and these sections form the center of $\mathcal{W}(M)$.

\subsection{Flat connections}

A connection in $\mathcal{W}(M)$ is a linear map

$$
\mathcal{D}: \Gamma(\mathcal{W}(M)) \rightarrow \Gamma\left(\mathcal{W}(M) \otimes T^{*} M\right)
$$


such that

$$
\mathcal{D}(a \circ b)=\mathcal{D} a \circ b+a \circ \mathcal{D} b, \quad \mathcal{D} f=d f, f \in C^{\infty}(M)[[h]] \subset \mathcal{W}(M)
$$

Any such a connection can be extended to the forms $\mathcal{W}(M) \otimes \Lambda T^{*} M$. Let $\nabla$ be a symplectic connection on $T^{*} M$ (i.e., $\nabla \omega=0$ and $\nabla$ is torsion free). Obviously, $\nabla$ can be extended to be a connection on $\mathcal{W}(M)$. Let $\Gamma^{\prime} \in \mathcal{W}(M) \otimes T^{*} M$. Then any connection in $\mathcal{W}(M)$ is of the form

$$
\mathcal{D} s=\nabla s+\frac{i}{h}\left[\Gamma^{\prime}, s\right]
$$

(the condition in (1.1.1) implies that any commutator is a multiple of $h)$.

Consider the symplectic form $\omega \in T^{*} M \otimes T^{*} M$. The first tensor factor of $\omega$ can be regarded as an element of $\mathcal{W}(M)$, and we get the form $\Sigma^{\prime} \in \mathcal{W}(M) \otimes T^{*} M$. Put $\Sigma=-\Sigma^{\prime}$.

The main point in the Fedosov construction is a flat connection $\nabla+\Gamma^{\prime}$ in $\mathcal{W}(M)$ such that

$$
\Gamma^{\prime}=\Sigma+\Gamma
$$

where $\Gamma=o(2)$, i.e., all summands in $\Gamma$ have their grading not less than 3 . The flatness means that the curvature form $\Omega=\mathcal{D}^{2}$ is a purely scalar form. This form is called the Weyl curvature. Such flat connections are called Fedosov connections. They do always exist and can be constructed by means of a certain iteration process [1]. Covariant constant sections of $\mathcal{W}(M)$ form a subalgebra $\mathcal{W}(M)_{\mathcal{D}} \subset \mathcal{W}(M)$, and the symbol map $\sigma: \mathcal{W}(M)_{\mathcal{D}} \rightarrow C^{\infty}(M)[[h]], s \mapsto \sigma(s)$ is invertible. Furthermore, the map $\sigma$ defines a one to one correspondence between the space of $C^{\infty}$-jets at a point $x$ and the fiber of $\mathcal{W}(M)$ at the same point (jet of a function $f$ goes into the section $\sigma^{-1} f$ restricted to $x$ ). We have obtained a one to one map

$$
\text { quant }: \mathcal{W}\left(T^{*} M_{x}\right) \rightarrow J_{x}^{\infty}(M)[[h]]
$$

Now we are in a position to construct a deformation quantization. Put

$$
f * g=\sigma\left(\sigma^{-1} f \circ \sigma^{-1} g\right)
$$

Note that the map quant defines an isomorphism between the Weyl Algebra and the jet algebra $J_{x}^{\infty}\left(\mathbb{A}^{h}\right)$ corresponding to our quantization. 


\subsection{Some formulas for symmetric Kähler manifolds}

In this subsection we shall establish some sign conventions and collect the identities for the curvature tensor that we will use in the sequel. All these identities are well known and some of them can be found in [6].

Let $M$ be a Kähler locally symmetric manifold with metrics $g$ and symplectic form $\omega$. Let $x_{0}$ be a point in $M$ and $e_{1}, e_{2}, \ldots, e_{n}$ be a unitary basis in $T_{\partial}^{*} M_{x_{0}}$. Then we have bases $\bar{e}_{1}, \bar{e}_{2}, \ldots, \bar{e}_{n} ; e^{1}, e^{2}, \ldots, e^{n}$, and $\bar{e}^{1}, \bar{e}^{2}, \ldots, \bar{e}^{n}$ in $T_{\bar{\partial}} M_{x_{0}}, T_{\partial}^{*} M_{x_{0}}$, and $T_{\bar{\partial}}^{*} M_{x_{0}}$ respectively. We have

$$
g=\sum e^{k} \otimes \bar{e}^{k} ; \quad \omega=\frac{1}{i} \sum e^{k} \wedge \bar{e}^{k} .
$$

The symplectic product $<,>$ on $T^{*} M$ is given by

$$
<e^{k}, \bar{e}^{j}>=-i \delta^{k j} ; \quad<e^{k}, e^{j}>=<\bar{e}^{k}, \bar{e}^{j}>=0 .
$$

Respectively, on $T M$ we have

$$
<e_{k}, \bar{e}_{j}>=-i \delta_{k j} ;<e_{k}, e_{j}>=<\bar{e}_{k}, \bar{e}_{j}>=0 .
$$

The hermitian connection $\nabla$ corresponding to $g$ is torsion free; therefore it is a symplectic connection and coincides with the Levi-Civita connection. Let $R\left(e_{i}, \bar{e}_{j}\right) e_{k}=R_{k l i j} e_{l}$. Since $R(X, Y)$ is antihermitian for real $X$ and $Y$, we have

$$
\overline{R_{k l p j}}=R_{l k j p}
$$

Using this, we find

$$
R\left(e_{p}, \bar{e}_{j}\right) \bar{e}_{k}=-R_{k l p j} \bar{e}_{l} .
$$

The Riemann identity $R(X, Y) Z+R(Y, Z) X+R(Z, X) Y=0$ yields

$$
R_{k l p j}=R_{p l k j}=R_{k j p l}
$$

The identity $\nabla^{2} R=0$ reads

$$
\sum_{k} R_{a k p j} R_{c d k b}-R_{k b p j} R_{c d a k}+R_{c k p j} R_{k d a b}-R_{k d p j} R_{c k a b}=0 .
$$

Let $R_{i j}=\sum_{k} R_{k k i j}$ be the Ricci tensor. Let us prove that

$$
\sum_{k} R_{a k} R_{c d k b}=\sum_{k} R_{k b} R_{c d a k}=\sum_{k} R_{c k} R_{k d a b}=\sum_{k} R_{k d} R_{c k a b}
$$


Contracting (1.4.3) with $\delta^{i j}$ and using (1.4.2), we get

$$
\sum_{k}\left(R_{a k} R_{c d k b}-R_{k b} R_{c d a k}+R_{c k} R_{k d a b}-R_{k d} R_{c k a b}\right)=0 .
$$

Contracting (1.4.3) with $\delta^{i d}$ and alternating $j$ and $b$ give

$$
\sum_{k}\left(R_{j k} R_{c k a b}-R_{b k} R_{c k a j}\right)=0,
$$

or

$$
\sum_{k} R_{j k} R_{c k a b}=\sum_{k} R_{b k} R_{c j a k}
$$

Taking the complex conjugate and using (1.4.1) lead to

$$
\sum_{k} R_{k j} R_{k c b a}=\sum_{k} R_{k b} R_{j c k a}
$$

Thus (1.4.4) follows from (1.4.5), (1.4.6), and (1.4.7).

\subsection{Fedosov connection for symmetric Kähler manifolds}

We will apply the Fedosov construction to a locally symmetric Kähler manyfold $M$ with the corresponding Kähler metrics $g$ and the symplectic form $\omega$. Our main goal is to construct a Fedosov connection on $M$. A direct application of the algorithm from [1] causes certain difficulties and, probably, can not be completed in a compact form. Fortunately, in our case, there exists another way to construct a Fedosov connection.

Consider the curvature tensor $R$ as an element of $T_{\partial}^{*} M \otimes T_{\partial}^{*} M \otimes$ $T_{\partial}^{*} M \otimes T_{\bar{\partial}}^{*} M$. We may consider the 2 -nd, the 3 -rd, and the 4 -th tensor multiples as elements of $\mathcal{W}(M)$, and we get a form $\Gamma \in \mathcal{W}(M) \otimes T^{*} M$. In a unitary basis $e^{1}, e^{2}, \cdots, e^{n}$ we have

$$
\Gamma=R_{k l p j} \bar{e}^{j} e^{k} \bar{e}^{l} \otimes e^{p}, \Sigma=\frac{1}{i} \sum e^{p} \otimes \bar{e}^{p}-\bar{e}^{p} \otimes e^{p},
$$

Set $\mathcal{D} s=\nabla s+\frac{i}{h}\left[\Sigma-\frac{1}{2 i} \Gamma, s\right]$.

Theorem 1.1. The connection $\mathcal{D}$ is flat, and the Weyl curvature of this connection is equal to $\omega$. 
Proof. For the sake of convenience set

$$
\delta s=\frac{i}{h}[\Sigma, s]=-\sum\left(\frac{\partial s}{\partial \bar{e}^{p}} \otimes \bar{e}^{p}+\frac{\partial S}{\partial e^{p}} \otimes e^{p}\right)
$$

and $\gamma=\frac{1}{2 i} \Gamma$. The curvature $\Omega$ equals $\Omega=\omega+r-\delta \gamma+\frac{i}{h}[\gamma, \gamma]$, where $r=(1 / i) R_{k l p j}\left(e^{k} \bar{e}^{l} \otimes e^{p} \wedge \bar{e}^{j}\right) \in \mathcal{W}(M) \otimes \Lambda^{2} T^{*} M$. Let us prove that $\Omega$ is a scalar and equals $\omega$. Note that $\delta \gamma-r=0$. Let us show that $[\gamma, \gamma]=0$. This reads as

$$
\begin{aligned}
& {\left[R_{k b i j} \bar{e}^{j} e^{k} \bar{e}^{b}, R_{c d a l} \bar{e}^{l} e^{c} \bar{e}^{d}\right]} \\
& \quad=4 h e^{c} \bar{e}^{j} \bar{e}^{b} \bar{e}^{d}\left(R_{k b i j} R_{c d a k}-R_{c k i j} R_{k d a b}\right) .
\end{aligned}
$$

Now, take (1.4.3) with $i$ and $a$ alternated and contract it with $4 h e^{c} \bar{e}^{j} \bar{e}^{b} \bar{e}^{d}$. We will get (1.5.1), which proves the theorem. q.e.d.

Set

$$
\theta^{p}=\bar{e}^{p}+\frac{1}{2} R_{k l p j} \bar{e}^{j} e^{k} \bar{e}^{l}
$$

Then

$$
\mathcal{D} s=\nabla s+\frac{1}{h} \sum\left[e^{p}, s\right] \otimes \bar{e}^{p}-\left[\theta^{p}, s\right] \otimes e^{p} .
$$

Let us construct explicitly the quantization map (1.3.1). Introduce in a neighbourhood of a point $x \in M$ the geodesic coordinates

$$
a^{1}, \bar{a}^{1}, \cdots, a^{n}, \bar{a}^{n}
$$

so that $\left.d a^{p}\right|_{x}=e^{p}$.

Theorem 1.2. We have

$$
\text { quant }(s)(a)=\sigma\left(e^{\frac{1}{h} \text { ad } \sum a^{p} \theta^{p}-\bar{a}^{p} e^{p}} s\right)
$$

where ad $x=[x, \cdot]$, and the exponent is treated as a formal series.

Proof. Consider a geodesic line $\gamma^{p}=\alpha^{p} t$. Let us transport the basis $e^{1}, \ldots, e^{n}$ along it, and write down the condition for a section $\tilde{s}(t)$ of $\mathcal{W}(M)$ to be a covariant constant along the geodesic line,

$$
\frac{d \tilde{s}}{d t}=\frac{1}{h} \sum\left[\alpha^{p} \theta^{p}-\bar{\alpha}^{p} e^{p}, \tilde{s}\right] .
$$

We have up to $o\left(t^{\infty}\right)$ that $\tilde{s}=e^{\frac{1}{h} \text { ad } \sum \alpha^{p} \theta^{p}-\bar{\alpha}^{p} e^{p}} \tilde{s}(0)$. Now it suffices to set $a^{p}=t \alpha^{p}$ and to calculate the symbol. q.e.d. 


\subsection{Quantization of Hamilton functions for infinitesimal symmetry transforms}

Consider a simply connected open subset $U$ in $M$. Infinitesimal symmetry transforms are Hamiltonian vector fields on $U$, which correspond to certain Hamilton functions. We will quantize these functions.

At each point $x_{0}$, let us define in the fiber $\mathcal{W}(U)_{x_{0}}$ a subspace $g_{x_{0}}$, generated over $\mathbb{C}$ by the elements $e^{i}, \theta^{i}, t_{i j}=\delta_{i j}+R_{k l i j} e^{k} \bar{e}^{l}$. Each of these subspaces forms a Lie algebra with respect to commutator $i / h[\cdot, \cdot]$. Indeed,

$$
\begin{aligned}
(i / h)\left[e^{p}, e^{j}\right] & =(i / h)\left[\theta^{p}, \theta^{j}\right]=0 ; \\
(i / h)\left[e^{p}, \theta^{j}\right] & =-i t_{j p} ; \\
(i / h)\left[e^{p}, t_{k j}\right] & =-i R_{l p k j} e^{l} \\
(i / h)\left[\theta^{p}, t_{k j}\right] & =i R_{p l k j} \theta^{l} .
\end{aligned}
$$

These identities follow from $(1.4 .3),(1.4 .2)$. The commutator $\left[t_{i j}, t_{k l}\right]$ can be obtained from the Jacoby identity. All these algebras are isomorphic to the compexified Lie algebra $g$ of symmetry transforms of $M$ and form a bundle $P$ which is preserved by the flat connection $\mathcal{D}$ and, therefore, is trivial over $U$ (because $U$ is simply connected). The space $\mathcal{F}$ of covariant constant sections of $P$ has dimension $\operatorname{dim} g$. Denote $\Pi=\sigma(\mathcal{F})$.

Proposition 1.3. The space $\Pi$ is closed with respect to the complex conjugation.

Proof. In each fiber $g_{x_{0}}$ of $P$ define a $\mathbb{C}$-antilinear map $J$ as follows:

$$
J\left(e^{p}\right)=\theta^{p} ; J\left(\theta^{p}\right)=e^{p} ; J\left(t_{p j}\right)=t_{j p} .
$$

One checks that $J$ is an $\mathbb{R}$-linear isomorphism of $g_{x_{0}}$ as a Lie algebra over $\mathbb{R}$. Using (1.5.2), we see that $J$ is a covariant constant. Note that $\sigma(J s)=\overline{\sigma(s)}$ for $s \in \mathcal{F}$, whence the proposition follows. q.e.d.

\section{Theorem 1.4.}

1. Let $s, t \in \mathcal{F}$, and $\{$,$\} be the Poisson bracket on M$. Then

$$
\sigma\left(\frac{i}{h}[s, t]\right)=\{\sigma(s), \sigma(t)\}
$$

2. Hamiltonian vector fields $X_{f}, f \in \Pi$ form the Lie algebra of infinitesimal local symmetries of $M$. 
Proof. 1. Let

$$
s=c_{s}^{r}(a, \bar{a}) e_{s}^{r}+d_{s}^{r}(a, \bar{a}) \theta^{r}+p_{s}^{r j}(a, \bar{a}) t_{r j} .
$$

Eq.(1.5.4) implies that

$$
\begin{aligned}
\left.\frac{\partial s}{\partial a_{k}}\right|_{a=0} & =\frac{1}{h}\left[\theta^{k}, s\right], \\
\left.\frac{\partial s}{\partial \bar{a}_{k}}\right|_{a=0} & =-\frac{1}{h}\left[e^{k}, s\right] .
\end{aligned}
$$

Hence,

$$
\begin{aligned}
\left.d \sigma(s)\right|_{a=0} & =\frac{1}{h} \sigma\left(\left[\theta^{k}, s\right]\right) e^{k}-\frac{1}{h} \sigma\left(\left[e^{k}, s\right]\right) \bar{e}^{k} \\
& =c_{s}^{k} e^{k}+\left.d_{s}^{k} \bar{e}^{k}\right|_{a=0}
\end{aligned}
$$

Since the origin $a=0$ can be chosen arbitrarily, this formula holds for every point.

Let $t=\sum c_{t}^{r}(a, \bar{a}) e_{t}^{r}+d_{t}^{r}(a, \bar{a}) \theta^{r}+p_{t}^{r j}(a, \bar{a}) t_{r j}$. Then

$$
\left.\{\sigma(s), \sigma(t)\}\right|_{a=0}=-c_{s}^{k} d_{t}^{k}+c_{t}^{k} d_{t}^{k}=\left.\frac{i}{h} \sigma([s, t])\right|_{a=0} .
$$

2. Due to Proposition 1.3, it is enough to consider $s \in \mathcal{F}$ such that $\sigma(s)$ is real. This means that $J s=s$ and that

$$
d^{k}=\overline{c^{k}}, p^{r j}=\overline{p^{j r}}
$$

Let $X_{\sigma(s)}$ be the Hamiltonian vector field corresponding to $\sigma(s)$. It suffices to prove that the map $\nabla X_{\sigma(s)}: Y \mapsto \nabla_{Y} X_{\sigma(s)} \operatorname{maps} \Gamma\left(T_{\partial} M\right)$ to $\Gamma\left(T_{\partial} M\right)$ and that it is an antihermitian map. Indeed, in this case $L_{X_{\sigma(s)}} Y=\nabla_{X_{\sigma(s)}} Y-\nabla_{Y} X_{\sigma(s)}$ belongs to $\Gamma\left(T_{\partial} M\right)$ if $Y \in \Gamma\left(T_{\partial} M\right)$ and

$$
\begin{aligned}
& L_{X_{\sigma(s)}}(Y, Z)-\left(L_{X_{\sigma(s)}} Y, Z\right)-\left(Y, L_{X_{\sigma(s)}} Z\right) \\
& \quad=\left(\nabla_{Y} X_{\sigma(s)}, Z\right)+\left(Y, \nabla_{Z} X_{\sigma(s)}\right)=0 .
\end{aligned}
$$

Eq. (1.6.5) implies that

$$
X_{\sigma(s)}=-i\left(c^{k} \bar{e}_{k}-d^{k} e_{k}\right) .
$$

Hence, 


$$
\begin{aligned}
\left.\nabla_{e_{t}} X_{\sigma(s)}\right|_{a=0} & =-\left.i\left(\frac{\partial c^{k}}{\partial a^{t}} \bar{e}^{k}-\frac{\partial d^{k}}{\partial a^{t}} e^{k}\right)\right|_{a=0} \\
& =i R_{t k r s} p^{r s} e^{k}
\end{aligned}
$$

We have used (1.6.5) and (1.6.1). We see that $\nabla X_{\sigma(s)}$ maps holomorphic fields to holomorphic fields. Since $p^{r s}=\overline{p^{s r}}$, the matrix $\left\|i R_{i k r s} p^{r s}\right\|_{i k}$ is antihermitian. q.e.d.

Now we just need to prove that all symmetry transforms are Hamiltonian fields corresponding to functions from $\Pi$. This immediately follows from the coincidence of dimensions $\operatorname{dim} g=\operatorname{dim} \Pi$.

\subsection{Darboux coordinates}

We will construct Darboux coordinates in a neighbourhood of a point $x_{0} \in M$ that are expressed in terms of functions from $\Pi$.

Denote

$$
\begin{gathered}
\xi^{p}=\text { quant } e^{p} \\
\eta^{p}=\text { quant } \theta^{p} \\
\tau_{p j}=\text { quant } t_{p j} .
\end{gathered}
$$

The system of variables (1.7.1)-(1.7.2) (which are functions from $\Pi$ ) is non-degenerate in a neighbourhood $V_{1}$ of $x_{0}$. The correspondence (1.7.1)-(1.7.3) between generating functions and sections from $P$ gives us a hint how to construct Darboux coordinates. Note that $\theta^{i}=\bar{e}^{i} / 2+$ $(1 / 2) t_{i j} \bar{e}^{j}$. Let us specify new variables $\lambda^{i}$ by means of the system of equations

$$
\eta^{p}=\lambda^{p} / 2+(1 / 2) \tau_{p j} \lambda^{j}
$$

This system is non-degenerate in a neighbourhood $V_{2}$ of $x_{0}$. Let us choose a neighbourhood $\mathrm{V}$ of $x_{0}$ so that $V$ be diffeomorphic to the ball and $V \subset V_{1} \cap V_{2}$.

Theorem 1.5. We have in $V$

$$
\begin{gathered}
\tau_{p j}=\delta_{p j}+R_{k l p j} \xi^{k} \lambda^{l} \\
\omega=\frac{1}{i} \sum d \xi^{p} \wedge d \lambda^{p} .
\end{gathered}
$$

Thus, $\xi^{p}, \lambda^{p}$ are Darboux coordinates in $V$. 
Proof. Let us prove (1.7.5). Consider the ideal $U \in C^{\infty}(V)$ generated by the functions

$$
\varphi_{p j}=\tau_{p j}-\delta_{p j}-R_{k l p j} \xi^{k} \lambda^{l}
$$

Suppose we have proven that all the functions

$$
\left\{\xi^{p}, \varphi_{j k}\right\},\left\{\eta^{p}, \varphi_{j k}\right\}
$$

are in $U$. Then we have for $\varphi_{p j}$ a system of linear partial differential equations

$$
\left\{\xi^{p}, \varphi_{j k}\right\}=a_{p j k l m} \varphi_{l m} ; \quad\left\{\eta^{p}, \varphi_{j k}\right\}=b_{p j k l m} \varphi_{l m} .
$$

This system is of the form

$$
\frac{\partial F_{p}}{\partial x^{j}}=c_{p j k}(x) F_{k}
$$

One easily checks that $\varphi_{j k}\left(x_{0}\right)=0$. The uniqueness theorem for systems of the form (1.7.8) yields that $\varphi_{j k}=0$ which proves (1.7.5).

Let us prove that the functions in (1.7.7) are in $U$. The congruence modulo $U$ will be denoted as usual by $\equiv$. Theorem 1.4 yields $\left\{\xi^{i}, \eta^{j}\right\}=$ $(i / h)$ quant $\left[e^{p}, \theta^{j}\right]=-i \tau_{j p}$. Compute the Poisson bracket with $\xi^{i}$ and both sides of the Equation in (1.7.4):

$$
\begin{aligned}
-i \tau_{j p} & =\frac{1}{2}\left\{\xi^{p}, \lambda^{j}\right\}+\frac{1}{2}\left\{\xi^{p}, \tau_{j k}\right\} \lambda^{k}+\frac{1}{2} \tau_{j k}\left\{\xi^{p}, \lambda^{k}\right\} \\
& =\frac{1}{2}\left(\delta_{j k}+\tau_{j k}\right)\left\{\xi^{p}, \lambda^{k}\right\}-\frac{i}{2} R_{l p j k} \xi^{l} \lambda^{k}
\end{aligned}
$$

hence

$$
\begin{aligned}
\frac{1}{2}\left(\delta_{j k}+\tau_{j k}\right)\left\{\xi^{p}, \lambda^{k}\right\} & =-i\left(\tau_{j p}-\frac{1}{2} R_{l p j k} \xi^{l} \lambda^{k}\right) \\
& =-i\left(\tau_{j p}-\frac{1}{2} R_{l k j p} \xi^{l} \lambda^{k}\right) \\
& \equiv-\frac{1}{2} i\left(\delta_{j p}+\tau_{j p}\right) .
\end{aligned}
$$

The matrix $\frac{1}{2} i\left(\delta_{j p}+\tau_{j p}\right)$ is invertible; therefore

$$
\left\{\xi^{p}, \lambda^{j}\right\} \equiv-i \delta^{p j} .
$$


Similarly, let us compute the Poisson bracket of $\eta^{j}$ and both sides of (1.7.4). We have

$$
\left\{\eta^{j}, \lambda^{p}\right\}+\tau_{p k}\left\{\eta^{j}, \lambda^{k}\right\}+\frac{i}{2} R_{j l p k} \lambda^{l} \eta^{k}=0 .
$$

Let us prove that

$$
\left\{\eta^{p}, \lambda^{j}\right\} \equiv-\frac{i}{2} R_{j l p k} \lambda^{k} \lambda^{l}
$$

If we formally substitute (1.7.11) into (1.7.10), we will have on the lefthand side a function from $U$ (to check it, it suffices to substitute for $\tau_{i j}$ the congruent functions from the right-hand side of (1.7.5) and to use (1.4.3),(1.4.2)). The matrix $\delta_{i j}+\tau_{i j}$ is invertible, hence the congruences (1.7.11) do hold. Using (1.7.9) and (1.7.11), one can prove by direct computation that functions in (1.7.7) are in $U$. Thus, we have shown that $U=0$ and that all congruences modulo $U$ are identities. Now (1.7.6) easily follows from (1.7.9). q.e.d.

The coordinates $\lambda^{p}$ have another nice property.

Theorem 1.6. The functions $\lambda^{p}$ are antiholomorphic.

Proof. Symmetry transforms are known to preserve the complex structure. On the other hand, the identities in (1.7.9), (1.7.11) imply that holomorphic functions of $\lambda^{1}, \ldots, \lambda^{n}$ go to holomorpic functions of $\lambda^{1}, \ldots, \lambda^{n}$ under symmetry transforms. Furthermore, the action of the symmetry group is transitive on $M$. Now the theorem follows from the fact that the differentials $d \lambda^{1}, \ldots, d \lambda^{n}$ are antiholomorphic at $x_{0}$.

q.e.d.

\section{Computation of trace density for symmetric Kähler manifolds}

\subsection{The trace density}

In this section we will recall the definition of the trace density and give some formulas for its computation.

Let $\mathbb{A}^{h}(M)$ be a deformation of $C^{\infty}(M)$. For any local chart $U$ on $M$ with Darboux coordinates $X^{1}, X^{2}, \ldots, X^{2 n}$ the algebra $\mathbb{A}^{h}(U)$ is isomorphic to the standart Weyl deformation $W(U)$ with the multiplication given by

$$
(f \circ g)(X)=\left.e^{-i h / 2 \tilde{\omega}^{p j} \partial / \partial u^{p} \partial / \partial z^{j}} f(u) g(z)\right|_{u=z=X},
$$


where as usual

$$
\tilde{\omega}^{i i+n}=-\tilde{\omega}^{i+n i}=1 ; \text { otherwise } \tilde{\omega}^{i j}=0,
$$

so that $\omega=\tilde{\omega}^{i j} d x^{i} \wedge d x^{j}$. Moreover, the isomorphysm

$$
\psi: \mathcal{W}(U) \rightarrow \mathbb{A}^{h}(U)
$$

can be chosen so that

$$
\psi(f)=f+\sum h^{k} U_{k} f
$$

where $U_{k}$ are differential operators of finite order [4].

For elements of the Weyl deformation with compact support $f \in C_{0}^{\infty}(U)$ the trace is well defined by the formula

$$
\operatorname{Tr} f=\frac{1}{(2 \pi h)^{n}} \int_{U} f \omega^{n} / n ! .
$$

This trace is transferred to $\mathbb{A}_{0}^{h}(U)$ by means of an arbitrary (infinitely differentiable and continuous in $h$-adic topology) isomorphism $W(U) \rightarrow$ $\mathbb{A}_{0}^{h}(U)$. Then the trace is extended to $\mathbb{A}_{0}^{h}(M)$ by means of a partition of unity, and thus the obtained trace does not depend on a partition neither on a choice of the isomorphisms.

There exists a unique function $\rho$ on $M$, which is the Laurent series with respect to $h$, such that $\operatorname{Tr} f=\int_{M} \rho f \omega^{n} / n !$. The function $\rho$ is called the trace density. One can easily show that $\rho=1 /(2 \pi h)^{n}+o\left(1 / h^{n}\right)$.

We need to modify this definition so as to express the trace density in terms of jets. According to the locality property of deformations the algebra $\mathbb{A}^{h}(M)$ can be restricted onto jets at a point $x_{0}$. The corresponding jet algebra $J_{x_{0}}^{\infty}[[h]]$ is isomorphic to the Weyl algebra $\mathcal{W}$ on $\mathbb{R}^{2 n}<y^{1}, \ldots, y^{2 n}>$ with symplectic scalar product $<y^{i}, y^{j}>=\tilde{\omega}^{i j}$ (see subsection 1.2). Our purpose is to express the trace density in terms of such an isomorphism. To obtain a reasonable formula we need to put some restrictions on these isomorphisms.

For further purposes we have to consider a wider class of coordinate systems. Let $x^{1}, \ldots, x^{2 n}$ be "almost Darboux coordinates" in a neighbourhood of $x_{0}$, i.e., $\left\{x^{p}, x^{j}\right\}=\tilde{\omega}^{p j}+o(1)$ (e.g. the normal geodesic coordinates on a symmetric Kähler manifold). We can identify $J_{x_{0}}^{\infty}[[h]]$ and $\mathcal{W}$ by means of the map

$$
\theta: f(x, h) \mapsto f(y, h) .
$$


After that, given an isomorphism $\varphi: \mathcal{W} \rightarrow J_{x_{0}}^{\infty}[[h]]$ we can construct a $\operatorname{map} \chi=\varphi \circ \theta: J_{x_{0}}^{\infty}[[h]] \rightarrow J_{x_{0}}^{\infty}[[h]]$. We want that $\chi$ were a differential operator. Let us specify what kind of operator $\mathrm{d} \chi$ should be. Consider the space $\mathcal{D}_{P}$ of differential operators that are polynomials in $x, \partial / \partial x$ with the natural grading $|x|=1,|\partial / \partial x|=-1$. Consider the completion $\mathcal{D}^{\prime}$ of $\mathcal{D}_{P}$ with respect to this grading. The action of operators from $\mathcal{D}^{\prime}$ on $J_{x_{0}}^{\infty}$ is well defined as well as the action of operators from $\mathcal{D}=\mathcal{D}^{\prime}[[h]]$ on $J_{x_{0}}^{\infty}[[h]]$. We require that $\chi \in \mathcal{D}$ and $\chi=1+O(1)+O(h)$, where $O(1)$ means sum of operators from $\mathcal{D}$ with their grading not less than 1. The isomorphisms $\varphi$ such that corresponding operators $\chi$ satisfy these two conditions are called suitable. Clearly, in this case, $\chi^{-1} \in$ $\mathcal{D}$. Note that the restriction to $J_{x_{0}}^{\infty}[[h]]$ of the operator $\psi$ satisfies this requirement. More precisely, let $X=X(x)$ be transition functions from the almost Darboux coordinates $x$ to Darboux coordinates $X$ such that $X=x+o(x)$. Then the formal series in $X, h$ can be viewed as elements of $\mathcal{W}$ (if one substitutes $y$ for $X$ ), and the corresponding isomorphism $\varphi_{\psi}: \mathcal{W} \rightarrow J_{x_{0}}^{\infty}[[h]]$ can be written as follows

$$
\begin{aligned}
\varphi_{\psi}: f(X) \mapsto g(X) & =(\psi f)(X) \mapsto \varphi_{\psi} f(x)=g(X(x)) ; \\
\chi_{\psi} & =\varphi \theta: f(x) \mapsto g(X(x)) .
\end{aligned}
$$

Here, all functions are considered as functions of $2 n$ variables rather than functions of point in $U$. Of course, there corresponds a function on $U$ to each of these functions but $F(x(P)) \neq F(X(P))$, where $P \in U$. Note, that the maps $f(x) \mapsto(\psi f)(x)$ and $g(x) \mapsto g(X(x))$ are operators from $\mathcal{D}$. Therefore, $\chi_{\psi} \in \mathcal{D}$. The condition $\chi_{\psi}=1+O(1)+O(h)$ can also be checked easily. The operator $\chi_{\psi}^{-1}$ looks as follows. Let

$$
h(X)=\psi^{-1} f(x(X)) .
$$

Then $\chi_{\psi}^{-1} f=h(x)$.

For an operator $D \in \mathcal{D}$ we can define the conjugate by the same formula as in the case of conventional differential operators: $\left(x^{\mu} \partial / \partial x^{\nu}\right)^{*}=$ $(-1)^{|\nu|} \frac{\partial}{\partial x^{\nu}} \circ x^{\mu}$ for multiindices $\mu$ and $\nu$. It can be easily checked that the conjugation is well defined on $\mathcal{D}$ but it depends on a choice of coordinates.

Now we are in a position to find the trace density.

Proposition 2.1. Let $\varphi$ be a suitable isomorphism. Then

$$
\rho\left(x_{0}\right)=\frac{1}{(2 \pi h)^{n}}\left(\chi^{-1}\right)^{*} 1 .
$$


Proof. First, let us prove the proposition for $\varphi=\varphi_{\psi}$. Let $V$ and $W$ be neighbourhoods of $x_{0}$ such that $\forall P \in V \exists ! Q \in W: x^{i}(P)=X^{i}(Q)$. Note that (2.1.1) specifies a map $\tilde{\chi}_{\psi}^{-1}: C^{\infty}(W) \rightarrow C^{\infty}(V)$, its restiction onto jets at $x_{0}$ is $\chi_{\psi}^{-1}$. Let $f$ be a function with supp $f \in W$. By the definition of the trace,

$$
\begin{aligned}
\operatorname{Tr} f & =\frac{1}{(2 \pi h)^{n}} \int_{W}\left(\psi^{-1} f\right)(x) d x^{1} d x^{2} \ldots d x^{2 n} \\
& =\frac{1}{(2 \pi h)^{n}} \int_{V}\left(\psi^{-1} f\right)(x) d x^{1} d x^{2} \ldots d x^{2 n} \\
& =\frac{1}{(2 \pi h)^{n}} \int_{V} h(x) d x^{1} d x^{2} \ldots d x^{2 n} \\
& =\frac{1}{(2 \pi h)^{n}} \int_{V} \tilde{\chi}_{\psi}^{-1} f d x^{1} \ldots d x^{2 n} \\
& =\frac{1}{(2 \pi h)^{n}} \int_{V} f\left(\tilde{\chi}_{\psi}^{-1 *} 1\right) d x^{1} \ldots d x^{2 n}
\end{aligned}
$$

where $h(x)$ is in (2.1.1). Therefore, $\rho\left(x_{0}\right)=\left(1 / h^{n}\right) \chi_{\psi}^{-1 *} 1$ because $\chi_{\psi}$ is a restriction onto jets of $\tilde{\chi}_{\psi}$. For arbitrary $\chi$ the map $\lambda=\theta \chi^{-1} \chi_{\psi} \theta^{-1}$ is an automorphism of $\mathcal{W}$. Any such an authomorphism is of the form $e^{i / h \text { ad } H}$ for some $H \in \mathcal{W}$ (see [4]). One can easily check that $\theta\left(\chi^{-1} \chi_{\psi}\right)^{*} \theta^{-1}=\lambda^{*}=e^{-i / h \text { ad } H}$. Note that $\lambda^{*} 1=e^{i / h \text { ad } H^{*}} 1=1$. Therefore, $\left(\chi^{-1} \chi_{\psi}\right)^{*} 1=1$ and $\chi^{-1 *} 1=\chi_{\psi}^{-1 *}\left(\chi^{-1} \chi_{\psi}\right)^{*} 1=\left(\chi_{\psi}^{-1}\right)^{*} 1$.

q.e.d.

Similarly one can prove the following modification of this proposition. Let $J$ be a volume density, i.e., $\omega^{n} / n !=J d x^{1} \wedge \ldots \wedge x^{2 n}$.

\section{Proposition 2.2.}

$$
\chi^{*}(\rho J)=\frac{1}{(2 \pi h)^{n}}
$$

\subsection{Exponents}

We need to operate with expressions of the form $e^{-k(x) / h}$ where $k(x)$ is a positively definite quadratic form. In this section we legalize these expressions and show how to apply them to the trace computation. The corresponding formula also expresses the trace density in terms of 
the isomorphisms of algebras of jets but it is not valid for all suitable isomorphisms. Thus, we have to define a narrower class of isomorphisms.

Consider the space $J_{x_{0}}^{\infty}\left[\left[h^{-1}, h\right]\right]$ which is a completion of the space of polynomials in $x^{p}, h, h^{-1}$ with respect to the grading $\left|x^{p}\right|=1,|h|=$ $2,\left|h^{-1}\right|=-2$. Define the space $\mathcal{U}$ as a completion of the space of differential operators which are polynomials in $x, \partial / \partial x, h, h^{-1}$ with the following grading on it: $|x|=1,|\partial / \partial x|=-1,|h|=2,\left|h^{-1}\right|=-2$. Then operators from $\mathcal{U}$ act on $J_{x_{0}}^{\infty}\left[\left[h^{-1}, h\right]\right]$ and for $U \in \mathcal{U}, f \in J_{x_{0}}^{\infty}\left[\left[h^{-1}, h\right]\right]$, we have $|U f|=|U|+|f|$ for the evaluations defined by the gradings.

For an $X \in \mathcal{U}$ one can correctly define an operator $e^{k(x) / h} X e^{-k(x) / h}$. To do it one suffices to notice that this conjugation is obviously defined for $X$ being a polynomial in $x, \partial / \partial x, h, h^{-1}$ and that it transforms polynomial operators into polynomial operators preserving the grading.

Similarly, for $f \in J_{x_{0}}^{\infty}\left[\left[h^{-1}, h\right]\right]$ one can define the integral

$$
\left.\int e^{-k(x) / h} f d x^{1} \ldots d x^{2 n} \in \mathbb{C}\left[h^{-1}, h\right]\right] .
$$

For $f$ being a polynomial on $x, h, h^{-1}$ we put

$$
\begin{aligned}
\int e^{-k(x) / h} f d x^{1} \cdots d x^{2 n} & =\int_{\mathbb{R}^{2 n}} e^{-k(x) / h} f d x^{1} \cdots d x^{2 n} \\
& :=\frac{1}{h^{n}} \int_{\mathbb{R}^{2 n}} e^{-k(y)} f_{0}(\sqrt{h} y) d y^{1} \cdots d y^{2 n},
\end{aligned}
$$

where $f_{0}(y)=(f(y)+f(-y)) / 2$ is the even part of $f$. This operation preserves the gradings and is therefore well defined on $\mathcal{U}$. We will apply it to the trace density computation.

Proposition 2.3. Let $\varphi$ be an isomorphism $\mathcal{W} \rightarrow J_{x_{0}}^{\infty}\left[\left[h^{-1}, h\right]\right]$ such that the corresponding differential operator $\chi=\varphi \circ \theta$ is suitable and belongs to $\mathcal{U}$. Then

$$
\begin{gathered}
\int e^{-k(x) / h} \rho(x)\left(e^{k(x) / h} \chi e^{-k(x) / h}\right) 1 J d x^{1} \ldots d x^{2 n} \\
=\frac{1}{(2 \pi h)^{n}} \int e^{-k(x) / h} d x^{1} \ldots d x^{2 n} .
\end{gathered}
$$


Proof.

Lemma 2.4 For any $D \in \mathcal{U}$ and $\rho \in J_{x_{0}}^{\infty}\left[\left[h^{-1}, h\right]\right]$ we have

$$
\begin{gathered}
\int e^{-k(x) / h}\left(e^{k(x) / h} D e^{-k(x) / h}\right) 1 \rho d x^{1} \ldots d x^{2 n} \\
=\int e^{-k(x) / h} D^{*} \rho d x^{1} \ldots d x^{2 n}
\end{gathered}
$$

Proof of Lemma. One suffices to check it for $\rho$ and $D$ being polynomials. In this case the integrals can be treated as conventional integrals over $\mathbb{R}^{2 n}$, whence the statement of Lemma.

To prove the proposition, let us substitute $\chi$ instead of $D$. Thus we have:

$$
\begin{aligned}
\int e^{-k(x) / h} & \rho(x)\left(e^{k(x) / h} \chi e^{-k(x) / h}\right) 1 J d x^{1} \cdots d x^{2 n} \\
& =\int e^{-k(x) / h} \chi^{*}(\rho J) d x^{1} \cdots d x^{2 n} \\
& =\frac{1}{(2 \pi h)^{n}} \int e^{-k(x) / h} d x^{1} \cdots d x^{2 n}
\end{aligned}
$$

by Propositon 2.2. q.e.d.

We will investigate the case of symmetric manifolds so that $\rho$ appeared to be a constant function on $M$. We can rewrite the above proposition for this case.

Corollary 2.5. If $\rho$ is a constant function, we have

$$
\rho^{-1}=\frac{\int e^{-k(x) / h}\left(e^{k(x) / h} \chi e^{-k(x) / h}\right) J d x^{1} \ldots d x^{2 n}}{\frac{1}{(2 \pi h)^{n}} \int e^{-k(x) / h} d x^{1} \ldots d x^{2 n}} .
$$

\subsection{Computation for symmetric manifolds}

We will apply the tools developed in the previous section to compute the trace density for the quantization which was constructed above for locally symmetric Kähler manifolds. Let us outline the ultimate result.

Let $\Theta$ be an integral purely antiholomorphic form defined in a neighbourhood of a point $x_{0}$ such that

$$
d \Theta=\omega-\frac{h}{2 i} R,
$$


where $R=R_{k k i j} e^{i} \wedge \bar{e}^{j}$ is the Ricci curvature or, which is the same, the curvature on $T_{\bar{\partial}}^{*} M$. To define $\theta$ uniquely, let us require that

$$
\Theta=\sum z^{p} \Theta^{p}
$$

for some holomorphic coordinates $z,\left.z\right|_{x_{0}}=0$ and some 1-forms $\Theta^{i}$. Let $\gamma_{x_{0} \rightarrow x}$ be a geodesic line from $x_{0}$ to $x$ in a small neighbourhood $U$ of $x_{o}$. Denote by $R_{x} x_{0}$ the reflection of $x_{0}$ with respect to $x$. Consider the 'action'

$$
S(x)=\int_{\gamma_{x_{0} \rightarrow R_{x} x_{0}}} \Theta .
$$

It can be easily seen that in geodesic coordinates $(a, \bar{a})$ in a neigbourhood of $x_{0}$, we have

$$
S=\frac{2}{i}|a|^{2}+s,
$$

where $s=o\left(|a|^{2}\right)$ as a formal series from $J_{x_{0}}^{\infty}[[h]]$. This means that $e^{-\frac{i}{h} s(x)} \in J_{x_{0}}^{\infty}\left[\left[h^{-1}, h\right]\right]$. Hence, we may define the integral

$$
\int e^{-\frac{i}{h} S(x)} \omega^{n} \stackrel{\text { def }}{=} \int e^{-2|a|^{2} / h} e^{-\frac{i}{h} s(a)} \omega^{n}
$$

as in (2.2.1).

\section{Theorem 2.6}

$$
1 / \rho\left(x_{0}\right)=\frac{\int e^{-\frac{i}{h} S(x)} \omega^{n} / n !}{\frac{1}{(2 \pi h)^{n}} \int e^{-2|e|^{2} / h} i^{n} d e^{1} d \bar{e}^{1} \ldots d e^{n} d \bar{e}^{n}}=2^{n} \int e^{-\frac{i}{h} S(x)} \omega^{n} / n !
$$

This section is completely devoted to a proof of this theorem. We will use Corollary 2.5. First of all we need an isomorphism $\varphi$ such that the corresponding operator $\chi$ belongs to $\mathcal{U}$. We claim that the isomorphism quant in Theorem 1.2 matches this requirement.

Proposition 2.7 Let $x^{p}+i x^{n+p} / \sqrt{2}=a^{p}, x^{p}-i x^{n+p} / \sqrt{2}=\bar{a}^{p}$ be geodesic normal coordinates in a neigbourhood of a point $x_{0} \in M$, and $\varphi=$ quant. Then the corresponding morphism $\chi=\varphi \circ \theta$ is suitable and can be expressed as a differential operator from $\mathcal{U}$.

Proof. Let $\mathcal{C}=\mathbb{C}[[a, \bar{a}, e, \bar{e}, h]]$ with the grading $|a|=|\bar{a}|=|e|=$ $|\bar{e}|=1,|h|=2$. We can decompose quant $=\sigma \circ \tau$ where $\tau: \mathcal{W} \rightarrow$ $\mathcal{C}, \tau(f)=e^{h^{-1} \text { ad }\left(\bar{a}^{p} e^{p}-a^{p} \theta^{p}\right)} f$, and $\sigma: \mathcal{C} \rightarrow J_{x_{0}}^{\infty}\left[\left[h^{-1}, h\right]\right]$ is the simbol 
map. Note that $|\sigma|=0$. Decompose $h^{-1}\left(\bar{a}^{p} e^{p}-a^{p} \theta^{p}\right)=A+B$, where $A=h^{-1}\left(\bar{a}^{p} e^{p}-a^{p} \bar{e}^{p}\right)$ and $B=h^{-1} a^{p} R_{k l p j} \bar{e}^{j} \bar{e}^{l} e^{k} / 2$. Then $|\operatorname{ad} A|=0$, $|\operatorname{ad} B|=2$. Therefore, we can use the perturbation theory formula

$$
e^{\mathrm{ad} A+\mathrm{ad} B} f=\sum_{n=0}^{\infty} \delta_{n} e^{\mathrm{ad} A} f=\sum \delta_{n} f(a+e, \bar{a}+\bar{e})
$$

where

$$
\delta_{0}=1, \quad \delta_{n}=\int_{0 \leq t_{1} \leq \cdots \leq t_{n} \leq 1} \text { ad } L\left(t_{1}\right) \ldots \text { ad } L\left(t_{n}\right) d t_{1} \ldots d t_{n}
$$

and $L(t)=e^{\operatorname{tad} A} B$. Note that $(\operatorname{ad} A)^{4} B=0$. Therefore, $L(t)$ is a polynomial in $t$, and ad $L(t)$ for any $t$ is a polynomial differential operator $\mathcal{W} \rightarrow \mathcal{C}$ with grading 2 . Hence, all $\delta_{n}$ are polynomial differential operators $\mathcal{W} \rightarrow \mathcal{C}$ with grading $2 n$. It easy follows that

$$
\Delta_{n}: f(a) \mapsto \sigma\left(\delta_{n} f(a+e, \bar{a}+\bar{e})\right)
$$

are polynomial differential operators

$$
J_{x_{0}}^{\infty}\left[\left[h^{-1}, h\right]\right] \rightarrow J_{x_{0}}^{\infty}\left[\left[h^{-1}, h\right]\right]
$$

with grading $2 n$. Finally,

$$
\chi=\text { quant } \circ \theta=\sum \Delta_{k}
$$

Thus, $\chi=$ quant $\circ \theta \in \mathcal{U}$ because $\left|\Delta_{k}\right| \rightarrow \infty$. To show that quant is suitable one suffices to check that $h$ only appears in the differential operator $\chi$ in positive degrees and that $\chi=1+O(1)+O(h)$. This easily follows from Theorem 1.2. q.e.d.

Put $k(e, \bar{e})=2|e|^{2}$. To calculate the trace density we need to calculate $\left(e^{2|a|^{2} / h} \chi e^{-2\left|a^{2}\right| / h}\right) 1$. To prove Theorem 2.6 it suffices to show that

$$
e^{2|a|^{2} / h} \chi e^{-2\left|a^{2}\right| / h} 1=e^{2|a|^{2} / h} e^{-\frac{i}{h} S(a)}
$$

As usual, let us write down an intuitively obvious expression for this quantity, explain how to understand this expression in strict terms of formal series, and prove that this expression does equal to $\left(e^{2|a|^{2} / h} \chi e^{-2\left|a^{2}\right| / h}\right) 1$. 
If $e^{-2|a|^{2} / h}$ were a usual function we would be able to write

$$
\left(e^{2|a|^{2} / h} \chi e^{-2\left|a^{2}\right| / h}\right) 1=e^{2|a|^{2} / h} \sigma\left(e^{\frac{1}{h} \operatorname{ad} \sum_{p}\left(a^{p} \theta^{p}-\bar{a}^{p} e^{p}\right)} e^{-2|e|^{2} / h}\right) .
$$

The last expression can be represented as a formal series in $a$ so that

$$
\left(e^{2|a|^{2} / h} \chi e^{-2\left|a^{2}\right| h}\right) 1=\sum_{\mu} a^{\mu} \sigma\left(D_{\mu} e^{-2|e|^{2} / h}\right),
$$

where $D_{\mu}$ are differential operators that are polynomials in $e, \bar{e}, \partial / \partial e, \partial / \partial \bar{e}$. Hence, $D_{\mu} e^{-2|e|^{2} / h}=P_{\mu}\left(e, \bar{e}, h, h^{-1}\right) e^{-2|e|^{2} / h}$ for some polynomials $P_{\mu}$. By definition, set

$$
\sigma\left(D_{\mu} e^{-2|e|^{2} / h}\right)=P_{\mu}\left(0,0, h, h^{-1}\right) \in \mathbb{C}\left[h^{-1}, h\right] .
$$

Thus, we have arrived at the following statement.

\section{Proposition 2.8}

$$
\begin{aligned}
\left(e^{2|a|^{2} / h} \chi e^{-2\left|a^{2}\right| / h}\right) 1 & =\sum_{\mu} a^{\mu} P_{\mu}(0) \\
& :=e^{2|a|^{2} / h} \sigma\left(e^{\frac{1}{h} \text { ad } \sum_{p}\left(a^{p} \theta^{p}-\bar{a}^{p} e^{p}\right)} e^{-2|e|^{2} / h}\right) .
\end{aligned}
$$

Hence, the right-hand side converges in $J_{x_{0}}^{\infty}\left[\left[h^{-1}, h\right]\right]$.

Proof. Any element of $J_{x_{0}}^{\infty}\left[\left[h^{-1}, h\right]\right]$ is a well defined formal series in $a$ with coefficients in $\left.\mathbb{C}\left[h^{-1}, h\right]\right]$ ( but degrees of $h$ may be no bounded uniformly for all coefficients of the series). Obviously, this formal series converges in $J_{x_{0}}^{\infty}\left[\left[h^{-1}, h\right]\right]$. Thus the expressions in the statement being proved can be regarded as formal series in $a$, and it suffices to prove the coincidence of these series. In other words, if an element $u$ from $J_{x_{0}}^{\infty}\left[\left[h^{-1}, h\right]\right]$ is equal to a formal series $v$ in $a$ in the space of formal series, then $v$ converges in $J_{x_{0}}^{\infty}\left[\left[h^{-1}, h\right]\right]$ and $u=v$ in $J_{x_{0}}^{\infty}\left[\left[h^{-1}, h\right]\right]$. Obviously, we only need to prove that the series coincide up to $o\left(|a|^{k}\right)$. The proof of Propositon 2.7 implies that

$$
\left(e^{2|a|^{2} / h} \chi e^{-2\left|a^{2}\right| h}\right) 1=e^{2|a|^{2} / h} \sum_{k=1}^{N} \sigma\left(\delta_{k} e^{-2|a+e|^{2} / h}\right)+o(N),
$$

where the symbol is calculated in the same way as in (2.3.5) (Recall that each $\delta_{k}$ is a polynomial differential operator). To obtain this relation, one needs to take (2.3.3) into account and to check that any operator 
$T$ that is a polynomial in $a, e, \bar{a}, \bar{e}, \partial / \partial e, \partial / \partial \bar{e}$, defines the operator $\tilde{T}$ : $f(a) \mapsto \sigma(T f(a+e))$ and $e^{2|a|^{2} / h} \tilde{T} e^{-2|a|^{2} / h} 1=e^{2|a|^{2} / h} \sigma\left(T e^{-2|e+a|^{2} / h}\right)$. Now it suffices to notice that

$$
\sum \delta_{k} e^{-2|a+e|^{2} / h}=e^{\frac{1}{h} \operatorname{ad} \sum_{p}\left(a^{p} \theta^{p}-\bar{a}^{p} e^{p}\right)} e^{-2|e|^{2} / h}
$$

because of the definition of $\delta_{k}$. q.e.d.

Further, we will transform the expression on the right-hand side of (2.3.6) so as to get (2.3.4). Note that the expression on the righthand side of (2.3.6) is a formal series in $a$. The proof of Propositon 2.8 yields that we only need to prove that the series (2.3.6) coincides with the formal series on the right-hand side of (2.3.4). Thus, we need not $J_{x_{0}}^{\infty}\left[\left[h^{-1}, h\right]\right]$ anymore. We will start with some standart facts about the action of polynomial operators on the vacuum projector $e^{-2|e|^{2} / h}$ in the Weyl algebra.

First, recall the definition of the Weyl ordering. Suppose we have a polynomial $P(e, \bar{e})$ in the Weyl algebra. Suppose to each $e, \bar{e}$ we put in correspondence some differential operator $\hat{e}, \hat{\bar{e}}$ respectively. Let $P$ be a monomial $\Pi_{k=1}^{l} t_{k}$, where all $t_{k}$ are equal to one of the elements from $\{e, \bar{e}\}$. Define

$$
P(\hat{e}, \hat{\bar{e}})=\frac{1}{l !} \sum_{\sigma \in S_{l}} \Pi \hat{t}_{\sigma(k)} .
$$

For an arbitrary polynomial, $P(\hat{e}, \hat{\bar{e}})$ is defined by linearity.

Proposition 2.9 Let $P$ and $Q$ be polynomials.

1. $e^{-2|e|^{2} / h} \circ P(e, \bar{e})=e^{-2|e|^{2} / h} \circ\{P(h \partial / \partial \bar{e}, \bar{e}) 1\}$ (the Weyl ordering is assumed);

2. $\sigma\left(P(e, \bar{e}, h) \circ e^{-2|e|^{2} / h} \circ Q(e, \bar{e}, h)\right)$ $=\sigma\left(e^{-2|e|^{2} / h} \circ Q(e, \bar{e}, h) \circ P(-e,-\bar{e}, h)\right) ;$

3. $\sigma\left(e^{-2|e|^{2} / h} \circ P(\bar{e}, h)\right)=P(0, h)$.

Proof. Straightforward computation using the Moyal product formula (1).

Remark. Note that the correspondence $\hat{e}=h \partial / \partial \bar{e}, \hat{\bar{e}}=\bar{e}$ used in Prop. 2.9,1 does not preserve the commutator. This is because the vacuum projector $e^{-2|e|^{2} / h}$ is multiplied from the right hand side, and if we consider operators acting on the functions from the right hand side, then $[h \partial / \partial \bar{e}, \bar{e}]=-h=[e, \bar{e}]$, and the commutators are preserved. Since 
we use the symmetric Weyl ordering, the operator $P(h \partial / \partial \bar{e}, \bar{e})$ applied from the left gives the same result as the one applied from the right.

\section{Proposition 2.10}

$$
\left(e^{2|a|^{2} / h} \chi e^{-2\left|a^{2}\right| / h}\right) 1=e^{2|a|^{2} / h} \sigma\left(e^{-2|e|^{2} / h} \circ e^{-(2 / h)\left(a^{p} \theta^{p}-\bar{a}^{p} e^{p}\right)}\right) .
$$

Proof. We will use the identity $e^{\text {ad } A} B=e^{A} B e^{-A}$ which allows us to rewrite (2.3.6) as follows:

$$
\begin{aligned}
&\left(e^{2|a|^{2} / h} \chi e^{-2\left|a^{2}\right| / h}\right) 1= e^{2|a|^{2} / h} \sigma\left\{\sum_{k}\left(\frac{\left(1 / h\left(a^{p} \theta^{p}-\bar{a}^{p} e^{p}\right)\right)^{\circ k}}{k !}\right)\right. \\
&\left.\circ e^{-2|e|^{2} / h} \circ \sum_{l}-\frac{\left(1 / h\left(a^{p} \theta^{p}-\bar{a}^{p} e^{p}\right)\right)^{\circ l}}{l !}\right\} \\
&=e^{2|e|^{2} / h} \sigma\left\{e^{-2|e|^{2} / h} \circ \sum \frac{\left(-2 / h\left(a^{p} \theta^{p}-\bar{a}^{p} e^{p}\right)\right)^{\circ k}}{k !}\right\}
\end{aligned}
$$

The last identity follows from Proposition 2.9,2. q.e.d.

Using Proposition 2.9,1,3 to substitute $h \partial / \partial \bar{e}$ for $e$ and to compute the symbol, gives

$$
\begin{aligned}
\left(e^{2|a|^{2} / h} \chi e^{-2\left|a^{2}\right| / h}\right) 1= & e^{2|a|^{2} / h} \exp \left\{-2 / h\left(a^{p} \bar{e}^{p}-h / 2 R_{p j} a^{p} \bar{e}^{j}\right)\right. \\
& \left.\left.+2 \bar{a}^{p} \partial / \partial \bar{e}^{p}+a^{p} R_{k l p j} \bar{e}^{j} \bar{e}^{l} / 2 \partial / \partial \bar{e}^{k}\right)\right\}\left.1\right|_{\bar{e}=0},
\end{aligned}
$$

where $R_{i j}=R_{k l i j} \delta^{k l}$ is the Ricci curvature. Thus, we arrive at the exponent of a first order differential operator, which we may use characteristics to compute. Substituting for $\bar{e}$ in this operator the antiholomorphic coordinates $\lambda$ yields an operator in a neighbourhood of $x_{0}$. It can be easily checked (using(1.7.9) and (1.7.11)) that this operator equals

$$
(2 / i)\left(X_{-\bar{a}^{r}} \xi^{r}+a^{r} \eta^{r}\right)_{\text {antiholom }}-p(x),
$$

where $X_{f}$ means the Hamiltonian vector field corresponding to $f$ and $p(x)=(2 / h) a^{r}\left(\lambda^{r}+h / 2 R_{i j} \lambda^{j}\right)$. Our vector field is tangent to a geodesic line (at) and has the constant length of $2|a|$ along this geodesic. This means that we need to determine $p(x)$ just on this geodesic and that

$$
\left(e^{2|a|^{2} / h} \chi e^{-2\left|a^{2}\right| / h}\right) 1=e^{-\int_{0}^{1} p(2 a t) d t}=e^{-\frac{1}{2} \int_{0}^{2} p(a t) d t} .
$$

Let us find $p(a t)$. 


\section{Proposition 2.11.}

$$
p(a t)=\left.\frac{i}{h} r_{X} \Theta\right|_{a t}
$$

where $X=(2 / i) X_{-\bar{a}^{r}} \xi^{r}+a^{r} \eta^{r}$ is the tangent field to the geodesic line and $\Theta$ is as in (2.3.1).

We need

\section{Lemma 2.12 .}

$$
\Theta=\frac{1}{i}\left(\xi^{k} d \lambda^{k}-\frac{h}{2} R_{p j} \xi^{p} d \lambda^{j}\right) .
$$

Proof. We have to show that the right-hand side $\Theta$ of (2.3.7) satisfies:

$$
\begin{aligned}
& d \Theta=\omega+(h / 2 i) R, \\
& \Theta \text { satisfies }(2.3 .2) .
\end{aligned}
$$

Let us prove (2.3.8). We have

$$
\begin{aligned}
d \Theta & =(1 / i)\left(d \xi^{k} \wedge d \lambda^{k}+\frac{1}{2} h R_{p j} d \xi^{p} \wedge d \lambda^{j}\right) \\
& =\omega+\frac{1}{2 i} h R_{p j} d \xi^{p} \wedge d \lambda^{j}
\end{aligned}
$$

The form $\frac{1}{i} R_{p j} \xi^{p} \wedge d \lambda^{j}$ coincides with the Ricci form at the origin. The Ricci form is invariant with respect to the symmetries. Therefore, if $\frac{1}{i} R_{p j} d \xi^{p} \wedge d \lambda^{j}$ is also invariant, then $R=\frac{1}{i} R_{p j} d \xi^{p} \wedge d \lambda^{j}$. Let us check the invariance.

$$
\begin{aligned}
\frac{1}{i} L_{\xi_{k}} R_{p j} d \xi^{p} \wedge d \lambda^{j}= & \frac{1}{i} R_{p j} d \xi^{p} \wedge d\left\{\xi^{k}, \lambda^{j}\right\}=0 . \\
\frac{1}{i} L_{\eta_{k}} R_{p j} d \xi^{p} \wedge d \lambda^{j}= & \frac{1}{i} R_{p j} d\left(\left\{\eta^{k}, \xi^{p}\right\}\right) \wedge d \lambda^{j} \\
& +\frac{1}{i} R_{p j} d \xi^{p} \wedge d\left(\left\{\eta^{k}, \lambda^{j}\right\}\right) \\
= & R_{p j} d\left(R_{m n k p} \lambda^{n} \xi^{m}\right) \wedge d \lambda^{j} \\
& -R_{p j} d \xi^{p} \wedge d\left(\frac{1}{2} R_{j m k l} \lambda^{l} \lambda^{m}\right) \\
= & R_{p j} R_{m n k p} \xi^{m} d \lambda^{n} \wedge d \lambda^{j} \\
& +R_{p j} R_{m n k p} \lambda^{n} \wedge d \xi^{m} \wedge d \lambda^{j} \\
& -R_{p j} d \xi^{p} R_{j m k l} \lambda^{l} \wedge d \lambda^{m} .
\end{aligned}
$$


The last expression vanishes due to (1.4.4).

Let us prove (2.3.9). It suffices to show that all $\xi^{k}$ belong to the ideal generated by $\overline{\lambda^{1}}, \ldots, \overline{\lambda^{n}}$. Since $\xi^{k}=\overline{\eta^{k}}$ (see (1.6.2)),

$$
\xi^{k}=\overline{\lambda^{k}}+(1 / 2) \overline{R_{p l k j}} \overline{\lambda^{j}} \overline{\xi^{p}} \overline{\lambda^{l}},
$$

and therefore $\xi^{k}$ belongs to the ideal. q.e.d.

Proof of the Proposition. Since $\Theta$ is purely antiholomorphic,

$$
\begin{aligned}
(i / h) i_{X} \Theta= & (i / h) i_{X_{\text {antiholom }} \Theta} \\
= & (i / h)\left(2 \bar{a}^{i} \partial / \partial \lambda^{i}\right. \\
& \left.-a^{k} R_{k l p j} \lambda^{j} \lambda^{l} \partial / \partial \lambda^{p},(1 / i)\left(\xi^{p} d \lambda^{p}+(h / 2) R_{p i} \xi^{p} d \lambda^{p}\right)\right) \\
= & (1 / h)\left(2 \bar{a}^{p}-a^{k} R_{k l p j} \lambda^{j} \lambda^{l}\right)\left(\xi^{p}+(h / 2) R_{p i} \xi^{p}\right) .
\end{aligned}
$$

Consider the difference

$$
\begin{aligned}
D= & (i / h) i_{X} \Theta-p=(i / h) i_{X} \Theta-\left(2 / h a^{s}\right)\left(\lambda^{s}+(h / 2) R_{s j} \lambda^{j}\right) \\
= & (2 / h)\left(\bar{a}^{s} \xi^{s}-\left(a^{k} / 2\right) R_{k l s j} \lambda^{j} \lambda^{l} \xi^{s}-a^{s} \lambda^{s}+(h / 2) R_{p s} \bar{a}^{s} \xi^{p}\right. \\
& \left.+(h / 4) R_{k l s j} R_{p s} \lambda^{j} \lambda^{l} \xi^{p} a^{k}-(h / 2) R_{s j} a^{s} \lambda^{j}\right) \\
= & (2 / h)\left(\bar{a}^{s} \xi^{s}-a^{s} \eta^{s}+(h / 2) R_{p s} \bar{a}^{s} \xi^{p}\right. \\
& \left.-(h / 2) R_{p s} a^{p}\left(R_{k l s j} \lambda^{j} \lambda^{l} \xi^{k} / 2+\lambda^{s}\right)\right) .
\end{aligned}
$$

Here we have used (1.4.4). Since $\bar{a}^{s} \xi^{s}-a^{s} \eta^{s}=0$ along the geodesic line, we have

$$
D=R_{p s} \bar{a}^{s} \xi^{p}-R_{p s} a^{p} \eta^{s} .
$$

At the origin $D=0$. Let us compute the derivative of $D$ along the geodesic line.

$$
\begin{aligned}
(i / 2) L_{X} D & =\left\{\bar{a}^{k} \xi^{k}-a^{k} \eta^{k}, R_{p s} \bar{a}^{s} \xi^{p}-R_{p s} a^{p} \eta^{s}\right\} \\
& =i \bar{a}^{k} a^{p} R_{p s} \tau_{s k}-i a^{k} \bar{a}^{s} R_{p s} \tau_{k p} .
\end{aligned}
$$

For the bracket $\left\{\xi^{s}, \eta^{j}\right\}$, see (1.6.1). Eq.(1.4.4) implies that $R_{p s} \tau_{k p}=$ $R_{k p} \tau_{p s}$. Hence, $L_{X} D=0$ and $D=0$. q.e.d.

Now, to prove (2.3.4) we only need to write

$$
\int_{0}^{2 t} p(a t) d t=\left.\int_{0}^{2 t} \frac{2 i}{h} i_{X} \Theta\right|_{a t} d t=\frac{2 i}{h} \int_{\gamma_{x_{0} \rightarrow S_{x} x_{0}}} \Theta,
$$

where $x=a$ and, hence, $S_{x} x_{0}=2 a$. 


\subsection{Computation for the projective spaces.}

In this subsection using our formula we compute the trace density for the projective spaces.

Let $\left(Z^{0}: Z^{1}: \ldots: Z^{n}\right)$ be homogeneous coordinates in $\mathbb{C} P^{n}$. We choose the point $(1: 0: \ldots: 0)$ as an origin and set $z^{s}=Z^{s} / Z^{0}$. We will work in some small neighborhood of the otigin. Then the simplectic form is

$$
\omega=(1 / i) \partial \bar{\partial} \log \left(1+|z|^{2}\right)
$$

where $|z|^{2}=z^{s} \bar{z}^{s}$. The Ricci form $R$ equals $-i(n+1) \omega$, and the integral form $(2.3 .1)$ is $\Theta=(1 / i)(1-h(n+1) / 2) \bar{\partial} \log \left(1+|z|^{2}\right)$. We denote $C=$ $(1 / i)(1-h(n+1) / 2)$. Any geodesic line passing through the origin is $z^{i}=a^{i} t$ for some constant $a^{i}$ and the distance from the origin to a pont $z$ is $\arctan |z|$. Compute the action. Let $T$ be a positive number, such that the distance from 0 to $T z$ is twice as much as the distance from 0 to $z$. Then

$$
\begin{aligned}
S(z) & =\int_{\gamma_{0 \rightarrow R_{z} 0}} C \bar{\partial} \log \left(1+|z|^{2}\right) \\
& =C \int_{0}^{T} d \log \left(1+|z|^{2} t^{2}\right)=C \log \left(1+|z|^{2} T^{2}\right) .
\end{aligned}
$$

We have $\arctan T|z|=2 \arctan |z|$ and

$$
S(z)=C \log \frac{1+|z|^{2}}{1-|z|^{2}}
$$

For the sake of convenience, let us compute

$$
\begin{aligned}
\frac{1}{\rho \int \omega^{n} / n !} & =2^{n} \frac{\int e^{-i S / h} \omega^{n}}{\int \omega^{n}} \\
& =2^{n} \frac{\int e^{-i C / h \log \left(\frac{1+|z|^{2}}{1-|z|^{2}}\right)} \frac{\Pi d z^{s} \wedge d \bar{z}^{s}}{\left(1+|z|^{2}\right)^{n+1}}}{\int \frac{\Pi d z^{s} \wedge d \bar{z}^{s}}{\left(1+|z|^{2}\right)^{n+1}}}
\end{aligned}
$$

This integral should be understood in the formal sence, explained above. But the integral makes sence if we take $h$ to be a small positive number and integrate over a small enough ball $|z|=\epsilon$. Thus the 
obtained values of the integral coincide with the integral understood formally up to $h^{\infty}$ for any $\epsilon$ small enough. Let $N=i C / h=1 / h-(n+1) / 2$. Then we can assume that $N$ is a large positive number. We have:

$$
\frac{1}{\rho \int \omega^{n} / n !}=2^{n} \frac{\int_{0}^{\epsilon}\left(\frac{1-R^{2}}{1+R^{2}}\right)^{N} \frac{R^{2 n-1}}{\left(1+R^{2}\right)^{n+1}} d R}{\int_{0}^{\infty} \frac{R^{2 n-1}}{\left(1+R^{2}\right)^{n+1}} d R},
$$

where $R=|z|$. Substitution $t=\frac{1-R^{2}}{1+R^{2}}$ yields

$$
\begin{aligned}
\frac{1}{\rho \int \omega^{n} / n !} & =2^{n} \frac{\int_{\delta}^{1} t^{N}(1-t)^{n-1} d t}{\int_{-1}^{1}(1-t)^{n-1} d t} \\
& =2^{n} \frac{\Gamma(N+1) \Gamma(n)}{\Gamma(N+n+1) 2^{n} / n} \\
& =\frac{\Gamma(N+1) \Gamma(n+1)}{\Gamma(N+n+1)} .
\end{aligned}
$$

This expression can be written as an asymptotic series in $h$. Furthermore, for integer $N$ we have:

$$
\operatorname{tr} 1=\rho \int \omega^{n} / n !=C_{N+n}^{n}=|\mathcal{O}(N)|,
$$

which implies that our trace density coincides with the one given by the index theorem [4].

\section{References}

[1] B. V. Fedosov, A simple geometric construction of deformation quantisation, J. Differential Geom. 40 (1994) 213-238.

[2] - Index Theorem for deformation quantization, Preprint.

[3] A. V. Karabegov, On deformation quantization on a Kähler manifold, connected with Berezin's quantization, to appear in Funct. Anal. Appl.

[4] R. Nest \& B. Tsygan, Algebraic index theorem for families, Adv. Math. 113 (1995) 151-205. 
[5] D. E. Tamarkin, Topological invariants of connections over symplectic manifolds, Funct. Anal. Appl. 29 (1995) 45-56.

[6] N. Kobayashi \& K. Nomizu, Foundations of differential geometry, Vols. I, II, Interscience, New York, 1963-1969.

Pennsylvania State University 